\title{
Functional Role of Fascia Enveloping Thigh and between Muscles in the Knee Extensor of Frog
}

\author{
Yoshiki ISHII, PT, PhD, Daisuke KASAMATSU, PT, Sinji KOMEI, PT, Tomoyuki FUKUDA, PT \\ Department of Physical Therapy, Faculty of Health Care Sciences, Himeji Dokkyo University \\ Masahiro SAKITA, PT, MS \\ Department of Physical Therapy, Faculty of Health Science, Kyoto Tachibana University \\ Nobuaki SASAI, PT, PhD \\ Department of Physiotherapy, Faculty of Health Science, Suzuka University of Medical science \\ Teizo TSUCHIYA, PhD \\ Department of Speech-Language Therapy, Faculty of Health Science, Teikyo Heisei University
}

Purpose: The present study was investigated to know the functional role of deep fascia enveloping thigh and fascia between muscles in vivo.

Methods: We measured the length-force relationship, in vivo, in whole muscle preparations in knee extensor, triceps femoris muscle (TFM), of the frog, Rana catesbeiana. TFM consists of three muscles, Rectus femoris muscle (RFM), vastus madialis muscle (VMM) and vastus lateralis muscle (VLM). The sciatic nerve was exposed at dorsal portion of the pelvis and all the branches of sciatic nerve except for the one innervating VMM and VLM were cut. Experiments were performed on two different preparations of TFM; in one preparation, surface of TFM was totally covered with fascia (CTF), in the second one, hamstrings was removed from TFM (CRH).

Results: Length-force curve located at the longer length in CRH than in CTF. Both the ascending and descending limbs in the curves were steeper in CTF than in CRH. In muscle length of TFM in the posture for Jumping, the isometric contractile force in $\mathrm{CRH}$ was about 10 percent lower than that in CTF.

Conclusions: The results in the present study indicate that the active force development in CTF is more efficient than in $\mathrm{CRH}$, suggesting that one of the functional roles of deep fascia and fascia between muscles is to produce higher active outputs by bundling each component muscle that has different contractile property, into one whole muscle. 